\title{
Research on the Construction of Information Sharing Platform Framework of Automobile Supply Chain from the Perspective of Blockchain Technology
}

\author{
Zhiping Lu ${ }^{1,2}$, and Fei Zeng ${ }^{1,2, *}$ \\ ${ }^{1}$ School of Economics and Management, Guangxi University of Science and Technology \\ ${ }^{2}$ Research Center of Guangxi Industry High-Quality Development, Donghuan Road, Liuzhou, Guangxi, \\ China
}

Keywords: Blockchain technology; Automobile supply chain; Information sharing

\begin{abstract}
As a decentralized distributed technology, the data network built by blockchain technology is maintained by all nodes in the system, which has the characteristics of decentralization, untamperability, transparency, security and so on. In recent time, the rise of this technology has attracted much attention from finance, health care, education, public management, etc. The organic combination of this technology and supply chain network brings new changes to the industrial situation. This paper is an attempt to discuss the coupling of blockchain technology to automobile supply chain for problems such as the opacity of information and the difficulty of information interaction in the current information sharing level of automotive supply chain. This study aims to build a database of automobile product information from supply to recovery and proposes a system design concept of information sharing platform. This research will provide new ideas for visualizing product data and improving system efficiency in automotive supply chain.
\end{abstract}

\section{Introduction}

Under the background of economic globalization, with the continuous improvement of the level of China's automobile manufacturing industry, the composition of automobile supply chain is getting increasingly more complex. However, the supporting automotive supply chain management has been a low level of information, information sharing efficiency and other problems [1]. With the characteristics like decentralization, traceability and untamperability, as well as its great impact on financial transactions and social life, blockchain technology has been paid more and more attention from governments, industries and research institutions [2]. How to effectively utilize the blockchain technology to establish a mutual trust and safe collaboration environment, so as to maintain the information communication among enterprises on each node, shorten the time limit of information transmission and simplify the process. As mentioned above, it is the hot spot and difficult point of automotive supply chain research.

Based on the analysis of the operation process of the automobile supply chain and the discussion of the coupling between the blockchain technology and the automobile supply chain. In this article an idea has been put forward to construct the information sharing platform framework of the automobile supply chain from the perspective of the blockchain.

\section{Features of Blockchain}

\subsection{Decentralization}

Blockchain is a peer-to-peer network structure composed of many nodes and does not depend on any central node. In the form of distributed records and storage, each participant can provide and store data on the chain at its node. Above, this form of joint management and maintenance by multiple participants guarantees the multi-party information sharing under the distributed system.

\subsection{Transparency}

The consensus algorithm that determines the bookkeeping right is public. Anyone can query the 
blockchain data and develop related applications through the public interface except that the private information of the parties to the transaction is encrypted, which makes the participants mutually support and independent from each other [3].

\subsection{Security}

Asymmetric cryptography algorithm is used to solve the security problem of user information in blockchain network. According to the principle of cryptography, the system generates both public and private keys. Any user can use the public key to encrypt the information to ensure the authenticity of the information. The private key is strictly kept secret, and only the owner of the information can use the corresponding private key to decrypt the information.

\subsection{Untamperability and traceability}

In the blockchain system using timestamp technology, each block contains the information about the previous block. This makes the blockchain save all the historical data from the original block to the present, and its chain structure ensures that the blockchain is an untampered and traceable system.

\section{Construction of Information Sharing Platform Framework for Automobile Supply Chain}

\subsection{Analysis of automobile supply chain}

Automobile supply chain is a network chain structure system with many participants, high density and complex structure. In practice, problems such as low information level, blocked information flow and low information transparency in automobile supply chain restrict the overall collaborative development of automobile supply chain [4].

\subsubsection{Opaque upstream and downstream information}

Due to the information asymmetry caused by different information management level and other factors, the upstream and downstream subjects of the automobile supply chain are often in a complex game relationship [5]. One of the parties to the transaction takes advantage of information to set up trading barriers to profit. Such pursuit of short-term self-interest maximization will lead to a vicious circle in the supply chain system, resulting in an increase in the overall cost, which is not conducive to the coordinated development of the industry.

\subsubsection{Difficulties in tracking illegal activities}

The production, storage and sale of automotive products on the supply network are invisible to consumers [6]. They obviously do not want to buy counterfeit products, but with the increasing globalized composition of the supply chain, such concerns among consumers will increase [7]. Regulators such as government departments have to pay high time and labor costs to track and locate products when certain links in the supply chain go wrong. However, once the effective supervision can not be carried out in time, it will lead to the decline of the credibility of government departments and other adverse consequences [8].

\subsection{3 "Bullwhip effect" occurs frequently}

Since information sharing cannot be effectively realized, in the process of information flow from the final client to the original supplier, the demand information is magnified step by step, forming a "bullwhip effect" [9]. Overstocking not only increases costs, but also brings risks and uncertainties, which in turn reduces economic benefits.

\subsubsection{Consumer demand cannot be fulfilled}

The serious obstacle of information interaction make it difficult for automobile manufacturers to get real feedback from users and exchange valuable suggestions with suppliers, thus making it difficult to respond quickly to changes in demand. And this phenomenon will have a huge impact on downstream marketing and customer service, causing a vicious circle [5]. 


\subsection{Model}

Relying on the alliance chain framework of blockchain technology, the information sharing platform framework of automobile supply chain is built by Figure 1. Its members include: raw material suppliers, component suppliers, automobile manufacturers, distributors, end users, recyclers, and government departments and other regulatory agencies.

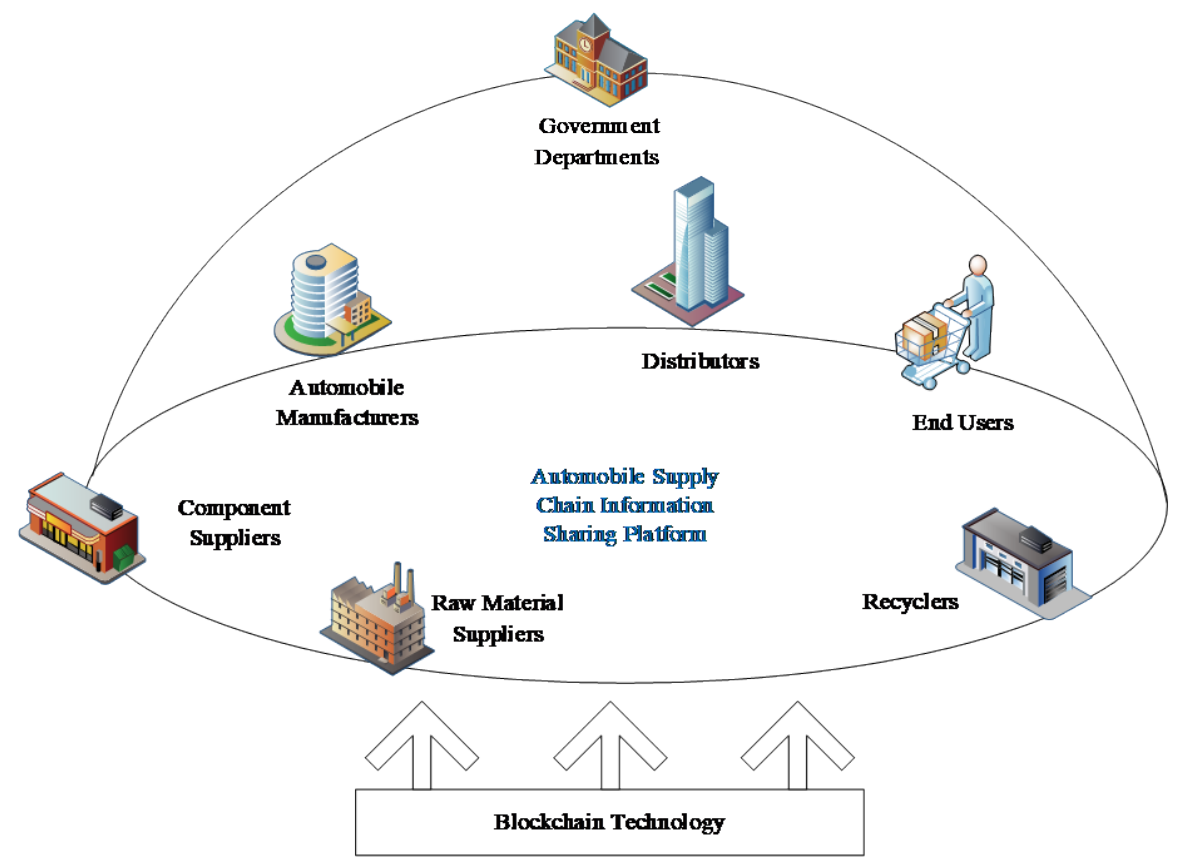

Figure 1 Frame Structure Drawing

The original supplier sets a unique identification code for each batch of materials and components, records the material information in the new block and uploads it to the information sharing platform built by the blockchain network. Based on the principle of asymmetric cryptography and the untamperability of blockchain, other members of the supply chain can check and trace all the materials in this batch. Even quickly locate items that may have been missed or changed during production or transportation. In this way, the information code that comes with the product correlates its identity in the physical world and the virtual world [10]. Thus, blockchain eliminates the need for a trusted central organization, and it allows consumers to check the ongoing process from raw materials to final sales. In addition, the timely disclosure and permanent preservation of information also facilitated the performance of responsibilities by regulatory agencies such as government departments.

Consumers directly show their willingness and preference on the platform, and distributors provide sales and inventory information, which is helpful for member enterprises of the automotive supply chain to timely adjust production strategies and make targeted sales plans. This enables the enterprises to meet demand while minimizing inventories. Besides, valuable user feedback will help stimulate the transformation and upgrading of the automobile industry from mass production to customized production.

Smart contracts cannot be changed once confirmed. Once the terms and procedures of the contract are satisfied, the system will automatically settle the transaction. Smart contracts play a supervisory role for both parties in this process [6]. The use of smart contracts can not only effectively save time and cost, but also improve the operation efficiency of the supply chain. It also provides the possibility for strengthening the partnership and cooperation consciousness among enterprises and promoting the coordinated development of industries. More importantly, in the era of economic globalization, the emergence of smart contracts has also provided great convenience for global procurement, which enables cross-regional transactions to be conducted in an open and transparent information environment. 


\subsection{System design}

Figure 2 shows the overall functional design of the system, including the base layer, the core layer, and the application layer.

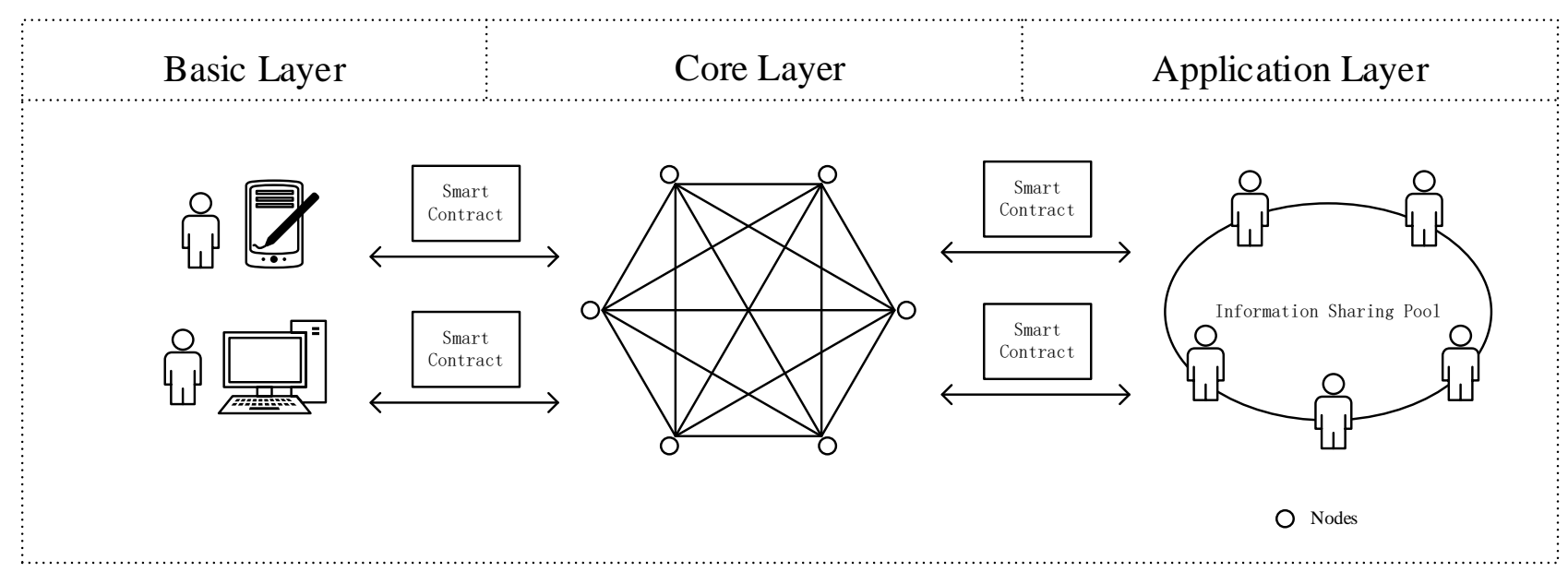

Figure 2 System design drawing

\subsubsection{Basic layer}

The basic layer is composed of the automobile supply chain information collection system. Each node in the automobile supply chain contains a large amount of data. Participants in each node collect and input information through terminal devices (such as mobile phones, computers, Internet of things devices, etc.) to form a distributed information collection system.

\subsubsection{Core layer}

The core layer is composed of blockchain data management network, and the collected information is stored in the blockchain data management network. Broadcast the data in the blockchain network, waiting for the nodes reach a consensus on the uploaded data, and then trigger the smart contract. Finally, the information is recorded on the block and saved in the distributed ledge.

\subsubsection{Application layer}

The application layer is a comprehensive application based on automotive supply chain information acquisition system and blockchain data management network. It mainly serves the relevant enterprises, regulatory departments and consumers in each link of the automotive supply chain. These users perform the corresponding functions by using functional modules provided by the platform, such as real-time monitoring of the supply chain by regulatory departments, traceability of products by consumers, management and sharing of their own information by enterprises, and so on.

The data information is acquired through the data acquisition terminal, and stored in the blockchain data management network after being verified by the smart contract. The application platform is mainly for related enterprises, consumers and regulatory departments. According to the different permissions given to each role by the blockchain, the node queries or submits the corresponding information through the smart contract. It not only ensures the openness and transparency of information, but also maintains a certain degree of privacy.

\section{Conclusion}

With the rapid development of economic globalization, the supply chain is characterized by multiple participants, wide geographical distribution and long time span. It hinders the sharing of information among members of the automotive supply chain. The introduction of blockchain technology has significantly improved the degree of information sharing, which is conducive to the 
integration of supply chain and the improvement of overall performance. It makes it possible to effectively solve the problem of trust between different subjects and promote the coordinated development of industry. Product traceability facilitates multiple parties to participate in supervision, so as to effectively prevent false transactions and vicious competition. Visualization of data can help enterprises optimize the process, strengthen the demand management, and further facilitate the prediction of the market to meet the personalized needs of customers.

However, blockchain technology also faces certain challenges, such as high energy consumption and high cost. With these problems expected to be solved, this technology will getting more important in the field of automobile supply chain.

\section{Acknowledgements}

The authors appreciate the valuable comments of anonymous referees. This paper is mainly sponsored by The Chinese National Social Science Foundation (No.18XGL006), the Guangxi Philosophy and Social Science Planning Research Project (No.15DGL004), the Innovation Project of Guangxi Graduate Education (No. YCSW2020223) and the Innovation Project of GXUST Graduate Education (No.GKJG201907) under Grant.

\section{References}

[1] Qian Zhang, Ju-hong Zhao. (2010) Discussion on the Development Status and Trend of Automobile Supply Chain Management in China. Macroeconomic Research, 4, 48-52(In Chinese).

[2] Qiu-ming Han, Ge Wang. (2018) A Review of Foreign Research on Blockchain Technology. Scientific and Technological Progress and Countermeasures, 35 (2), 154-160(In Chinese).

[3] Yadav, S., Singh, S.P. (2020) Blockchain Critical Success Factors for Sustainable Supply Chain. Resources, Conservation and Recycling(online).

[4] Hui-qin Yang, Lei Sun, and Xi-chao Zhao. (2018) Construction of Mutual Trust and Win-win Supply Chain Information Platform Based on Blockchain Technology. Scientific and Technological Progress and Countermeasures, 35 (5), 21-31(In Chinese).

[5] Ying-hao Qin et al. (2019) Blockchain Wave: Connection Technology and Applications. Beijing: Machinery Industry Press. pp. 63-73(In Chinese).

[6] Saberi, S., Kouhizadeh, M., Sarkis, J., and Le-jia Shen. (2019) Blockchain Technology and its Relationships to Sustainable Supply Chain Management. International Journal of Production Research, 57 (7), 2117-2135.

[7] Francisco, K., and Swanson, D. (2018) The Supply Chain Has No Clothes: Technology Adoption of Blockchain for Supply Chain Transparency. Logistics, 2 (1), 2-15.

[8] Ivanov, D., Dolgui, A., and Sokolov, B. (2019) The Impact of Digital Technology and Industry 4.0 on the Ripple Effect and Supply Chain Risk Analytics. International Journal of Production Research, 57 (3), 829-846.

[9] Zheng-jia Zhao, Zhi-hua He. (2018) Research on Supply Chain Inventory Management and its Relationship with Performance in Automobile Manufacturing Enterprises. Industrial Engineering and Management, 23 (2), 98-106(In Chinese).

[10] Abeyratne, S. A., and Monfared R. P., (2016) Blockchain Ready Manufacturing Supply Chain Using Distributed Ledger. International Journal of Research in Engineering and Technology, 5(9), $1-10$. 